\title{
Onychophagia (Nail Biting): an overview
}

\author{
Javed Ather Siddiqui ${ }^{1}$, Shazia Farheen Qureshi ${ }^{2}$ \\ ${ }^{1}$ Psychiatrist, Department of Psychiatry, Mental Health Hospital, Taif \\ ${ }^{2}$ Psychiatrist, Department of Psychiatry, Mental Health Hospital, Taif \\ Corresponding author: Javed Siddiqui \\ Email - javedsiddiqui2000@gmail.com
}

\begin{abstract}
Onychophagia (NB) is a self-grooming behavior, and a chronic habit of nail biting also called body focused repetitive behaviors (BFRBs). It is disorders in which the individual damages his or her appearance or causes physical injury. It may lead to significant physical and psychosocial problems, also having a negative impact on quality of life. It is commonly seen in children and young adult. The present paper focuses on the classification, etiology, epidemiology, and complications. It also throws lights on prevention, prognosis and management of NB.
\end{abstract}

Keywords: Onychophagia, nail biting, a body focused repetitive behaviors, self-grooming behaviors

(Paper received $-29^{\text {th }}$ November 2019, Peer review completed $-15^{\text {th }}$ February 2020)

(Accepted $-15^{\text {th }}$ February 2020)

\section{INTRODUCTION}

Onychophagia also called nail biting (NB), is a common but unresolved problem. It is a pathological oral habit in which a person puts as well as bites one's nails and finger tips. Onychophagia is a Greek word, onycho means finger nail or toe nail and phagia is to eat or consume. In such a condition, initially, actions are often performed to decrease a sense of built up tension and later often lead an immediate sense of relief and satisfaction. It is poorly understood, misdiagnosed, and is an undertreated group of disorders as well as is a less published area in psychiatry and dermatology [1].

It is a stress relieving oral habit, commonly occurs in children and young adults [2]. It is often seen when the affected person is stressed, frustrated, nervous, embarrassed, hungry or when bored in situations; nailbiting leaves the person feeling powerless, ashamed, and depressed. Onychophagia is a behaviour with an obsessive-compulsive spectrum disorder. It is often neglected as a condition in clinical practice and it may lead to negative effects on quality of life and results in physical as well as psychosocial damage. It is also considered as a self-mutilative automatic behaviour [3-4].

This self-grooming behaviour may lead to physical injury and mental health conditions such as serious emotional distress, anxiety and depression. Initially, NB can start because of anxiety, severe stress, or tension and then it converts into a habit; it persists even though the stress or anxiety is disappearing. If it is ignored and not treated properly in childhood, most likely it continues into adulthood as well. It occurs in 20 to 33 percent children and 45 percent in teenage group [5-7]. By the age of 18 years, the frequency of nail-biting decreases; however, it may persist in some adults [8].

It is often overlooked due to its benign nature. It became severe when the habit gets worst and destructive, leading to repeated infections due to which it gets difficult to use hands from routine work. Onychophagia is an essential terminology of nail tic disorders. Tic is a persistent, recurrent or repetitive behavioural trait and if involved in nail unit, it is called nail tic disorders. It is one of the misdiagnosed disorders that is 
poorly understood. People engaging in nail biting might have certain personality traits which may be referred to as a sign of perfectionist personality, and these traits point to perfectionism. Research suggests, NB is the perfectionist trait and it can be an underlying cause of these behaviours and perfectionism. Researchers said that boredom and frustration, these two traits come to the surface quickly with a perfectionist personality [9].

In the Diagnostic and Statistical Manual of Mental Disorders Fifth Edition (DSM-5), NB is classified as an "Other specified obsessive-compulsive and related disorder" with specification of "body-focused repetitive behaviour (BFRBs)," whereas International Statistical Classification of Diseases and Related Health Problems ICD-10 classifies the practice as "other specified behavioural and emotional disorders with onset usually occurring in childhood and adolescence."

Nail biters can also be called pathological groomers, but psychiatry makes changes the way that it thinks about pathological grooming. These changes are reflected in the American Psychiatric Association's Diagnostic and Statistical Manual of Mental Disorders as an obsessive-compulsive and related disorder. NB has been receiving growing scientific attention. One study claimed that NB is considered an involuntary behaviour with close to 70 percent people reporting tension before the behaviour and 40 percent reporting a feeling of temporary pleasure after the nail had been bitten. Many nail biters feel depersonalization during the episode.

\section{Classification of Nail Biters}

There are different references to nail biting and they are classified as -

- Nail biters that bite without realizing.

- Nail biters that bite to control anxiety.

- Attention seeking Nail biters.

- Nail biter to control aggression by self-injurious gesture.

- Nail biting that is a part of Obsessive-compulsive disorder spectrum.

- Nail biting which in DSM-5 is called; other specified obsessive-compulsive and related disorder.

- Nail biting which in ICD-10 is called; other specified behavioural and emotional disorders with onset usually occurring in childhood and adolescence.

- Nail biters who are also classified as Pathological and Non-pathological NB.

\section{Nail biting as a Psychiatric disorder}

There are two psychiatric disorders, such as Onychophagia and Onychotillomania that are related with NB. Chronic biting of nail is called Onychophagia whereas chronic picking and manicuring of nails is known as Onychotillomania.

\section{AETIOLOGY}

There are lot of controversies about the causes of NB so it is debatable regarding a habit and the psychodynamics of the same. NB is very extensive phenomenon and it is very difficult to break the practice. Most of the nail biters do not even notice when they are biting their nails, it feels like routine and without knowing they become addicted to the nail biting. NB evolves during teenage period because they experience changes and complexity of emotions and stress, if unable to face challenges and handle emotions, it leads to the development of nail biting. The basic aetiology of NB is difficult to determine, it is a learned behaviour from family members.

Psychotherapists have studied several theories on NB. Sigmund Freud criticizes it on arrested psychosexual development, at the oral stage. Typical to Freudian theories, oral fixation is linked to myriad causes, such as under-feeding or over-feeding, breast-feeding too long or problematic relationship with your mother. Some researchers have theorised about NB as being a sign of self-hostility and it result in selfmutilation or nervous anxiety. A poor self-image, a deep-seated guilt or anxiety over an unresolved problem is often a reason for compulsive nail biting. Over-stimulation, stress and excitement, understimulation or inactivity and boredom often act as triggers to this compulsive behaviour. While some 
studies said it is due to behavioural problems [10], other theories propose NB as a symptom of anxiety or a nervous habit [11]. Some other causes are a divorce in the family, a new school and psychosocial causes. Whereas in some conditions, NB is induced by low self-esteem, high anxiety, stress, loneliness, nervousness, or boredom and imitation of other family member; some condition like emotional and psychological issues also lead to NB. Researcher also say that anxiety in children with NB is not a trait; it is a state, the trait which is associated with NB is oral aggression [12].

Some studies said that NB is due to heredity predisposition. The children whose parents bite their nails have higher incidence than non-biters; 60 percent versus 15.5 percent. Concordance rates has been found to be higher in monozygotic twins than dizygotic twins; 66 percent versus 34 percent. Recent studies did not support the anxiety theory to NB, but supported that two of the largest contributing factors to NB were boredom and frustration. Some researchers accept that it is a result of a delay or dysfunction in the oral stage of psychological development. One more cause is imbalance in the neurotransmitters present in the brain accounting for a self-mutilating behaviour, but there is no evidence to support this claim.

\section{EPIDEMIOLOGY}

NB does not start before the age of three years, the frequency of NB increases from childhood to adolescence, and then decrease in adulthood [5]. Studies have said that nail biting occurs in children between ages 7 and 10 years which is 28 percent and 33 percent respectively, but nail-biting occurs most often during puberty, around 44 percent of adolescents, and 19 to 29 percent of young adults bite their nails [13]. Later at the age of 18 years, the frequency of nail-biting decreases. Only a small number of older adults bite their nails and they stop biting their nails on their own by age 30 . Some studies claim that NB is an age- related problem, and its frequency decrease with the increase of age [14]. NB is noticed significantly higher in boys than girls. If one of the members of family is a nail biter, then genetic chances of NB in children may be as high as 36.8 percent. One study said that in India its prevalence rate is 12.7 percent and girls are more affected than boys.

\section{Why people bite their nails?}

- Nervousness: When you are tensed and stressed, have lots of problems, so you may feel anxious and you are more likely to bite your nails. Biting nails has a calming effect on the nervous system.

- Imitation: Role models of children are their elders such as parents, and they are used to copying their adults whether it is a good or bad habit.

- Emotions: Emotions plays an important role in NB. Shyness and low self-esteem, as well as the pain caused by many reasons such as divorce or the death of a loved one can cause people to bite their nails.

- Boredom: If persons feel bored, are not doing anything in particular, are inactive- these could be some common reasons to bite their nails.

- Perfectionism: If children find they are imperfect in specific goal, they automatically try to fix them by nail biting.

- Psychosomatic: Particularly seen in aggressive families.

\section{PSYCHIATRIC DISORDERS ASSOCIATED WITH NB}

NB is not always an isolated problem but is also associated with other psychopathological symptoms or mental disorders. NB is associated with obsessive-compulsive spectrum disorder and this type of disorder has recently ben been called 'Body Focused Repetitive Behaviour'.

There are some underlying conditions associated with psychiatric disorders in children who act on the urge to bite their nails and suffer from attention deficit hyperactivity disorder (74.6 percent), oppositional defiant disorder (36 percent), and separation anxiety disorder (20.6\% percent). Other co-morbid disorders include enuresis (15.6 percent), tic disorder (12.3 percent) and also forms of obsessive-compulsive disorder (11.1 percent), major depressive disorder (6.7 percent), mental retardation ( 9.5 percent), and pervasive developmental disorder (3.2 percent) may be seen comorbid with NB [15]. Some conditions like 
generalized anxiety disorder and panic disorder have also been reported. Nail biting commonly co-occurs in 28.6 percent psychiatric problems among children and adolescents with Tourette syndrome. Stereotypic behaviour problems are also associated with NB in children; the commonly co-occurring stereotypic behaviours were lip biting (33.3 percent) and head banging (12.7 percent) [15]. Another study reported that 70 percent of individuals with hair pulling habit associated with other stereotypic behaviours, of which skin-picking and nail-biting were the most common ones. It is sometimes also proposed that a person with NB has higher obsessive-compulsive behaviours.

\section{COMPLICATIONS OF NAIL BITING}

Many complications are associated with NB. Most of the cases of NB are directed to cosmetics purpose [16]. Social complications of NB are humiliation, emotional suffering, and social impairment and if it is neglected and not treated then condition deteriorates. An individual's mental health as well as physical health are affected due to NB. Nail biting may actually be harmful to one beyond the emotional effects. If left untreated, severe onychophagia can lead to dysmorphic dental problems, including malocclusion painful blisters, abscesses and many more. In chronic nail biter shortening of nails that occur due to disfiguring behaviour [17]. In NB, bleeding occurs around the nail that causes paronychia, and leads to secondary bacterial infections such as onychomycosis and paronychia. Nail biting infection also spreads to the mouth that includes potentially pathogenic bacteria like Salmonella and E. coli. These bacteria easily transfer into the mouth and the rest of the body, where they may lead to infections and herpetic whitlow transmitted from oral herpes lesions. Warts on fingers of nail biters caused by human papilloma virus are common among chronic nail biters. These warts can easily transfer to mouth and lips as bite the nails. Rarely osteomyelitis of the phalanges and scarring leading to keloid formation can also occur in nail biters. Severe nail biting can produce dental problems, these are malocclusions, and small cracks or fractures at the edges of the incisors, it also harmful to the gingiva that leads gingivitis or abscesses [18]. Also due to biting, infections spread to abdomen, and that can lead to an increased risk for pinworm and giardia infections of the gastrointestinal tract [19]. Thus, NB leads to following complications:

1. Social and Psychological Complications: humiliation, emotional suffering, and social impairment.

2. Physical Complications: Malformed nails, Infection of the nail and surrounding soft tissue; increased risk of parasitic infections, Stomach infections due to swallowing nail particles and dirt; pain in the temporomandibular (TMJ) joint or jaw joint, injury to the gums, paronychia, selfinflicted gingival injuries, secondary bacterial infections, osteomyelitis.

\section{When to worry about Nail Biting}

Severe nail biting is a warning sign of an excessive anxiety and significantly impairs quality of life, that's when nail biting should be examined by psychiatrists. Psychiatric evaluation of co-occurring psychopathological symptoms in patients, especially those with chronic, severe or complicated onychophagia, may be helpful in making a choice of individual therapy. If severe NB is left untreated it can lead to psychological as well as physical complications.

\section{PREVENTION FROM NAIL BITING}

Nail biters can avoid biting nail need by focusing on strategy and some effort. Anxiety reduction techniques or routine preventive strategies are helpful to prevent yourself from nail biting. There are some measures that may be helpful, these are:

- Always keep your hand and mouth busy: to keep the hands busy doing something else, such as playing music, practicing sport activities, or spend more time with your friends. During the stressful situation or whenever alone chew the gum and stay active so biter will not put attention to put his finger in mouth. 
- Follow the Replacement habit: Nail biter should replace their habit with something more effective, for that reason biter must find fidgeting tools like spinner and drawing or writing or squeezing a stress ball or Silly Putty, when you find yourself biting your nails.

- Tell people to point it out: To request your close, near and dear people and those understand you instead laughing or criticizing on you. Tell them to point it out to you whenever you were put to your finger in mouth.

- To set up alarm reminder: If biter will bite nails at particular times of a day or places, they can set up an alarm that sends off reminder to the biter not to bite.

- To put something on your hands or on finger: If you put irritant over fingers to avoid it from biting you can prevent bite. One could also apply olive oil to nails, it softens them and makes them less appealing.

- Get regular manicures: Keep your nails trimmed and filed, to keep your nails looking attractive may make you less likely to bite them. Alternatively, you can also cover your nails with tape or stickers.

- To cover finger with bitter taste: There are some nail polishes of bitter taste available in market so that the biter can paint it on finger, and the bad taste prevents one form biting. You can put denatonium benzoate on your fingers, it is a bitter chemical compound. The bitter flavour discourages the nail-biting habit.

- To wear gloves: It may look silly, but biter gets away from biting, similar effect can be seen with putting stickers or adhesive bandages on finger, whenever possible to remind the biter not to bite.

- To relieve stress: Stress management can help to deal with Nail-Biting episodes and reduce its frequency so try stress-management techniques if one bites their nails when anxious or stressed. Stress is one of the factors behind the urge to bite nails. Incorporating activities such as meditation, yoga, or other forms of relaxation are good examples to relieve stress.

- To follow Support groups: Support groups play an important role and it can be established to help to cope with the symptoms and improve the quality of one's life.

- Support and encouragement from loved one: Family members, loved one and close friends can provide non-judgmental support and encourage their loved ones to seek early treatment for this potentially serious condition.

\section{PROGNOSIS}

The prognosis and its treatment response always depend on the severity of the signs and symptoms of nailbiters, and other factors. The prognosis of NB is generally good with proper treatment. A better understanding of the situation helps to achieve best results.

\section{MANAGEMENT OF NAIL BITING}

The main goal of management of NB is to motivate the patient and provide emotional support and to relieve stress that may help to defeat the nail biting. In mild cases, nail biting is not a distressing condition and always improve on its own, even though it needs more attention, love, affection, and comprehension; these are suitable to stop the bad habit. The best way to manage a nail biting is to make good habits, educate them, to develop awareness, to give emotional support and encouragement [20]. Detailed history including psychosocial aspects and a thorough physical examination are important, if it is associated with other comorbid disorders. In most of the cases of NB, it looks like a cosmetic issue, so no treatment is required. But, severe cases of NB need pharmacological treatment associated with non-pharmacology treatment such as psychotherapy. Anti-depressant is commonly used for treatment of NB such as fluoxetine [21] and clomipramine [22] but is limited in use. Best way to manage a nail biter is to educate them, to develop good habits, develop conscious awareness for effective results.

Emotional support and encouragement are the one of the best methods and should be supplemented during treatment. Avoid nagging and punishing to nail biter, it is not always useful. Always focus on 
efforts to build up the child's self confidence and self-esteem. In some cases nail biter needs cognitive behavioural techniques for their behaviours; these are habit reversal techniques and stress-management techniques. Nail biters bite the nail because they are anxious or stressed. Educate the affected children along with their parents, siblings, and teachers as a part of the treatment. Behavioural modification techniques, positive reinforcements, and regular follow-ups are important key factors for the treatment of nail biting. The punishment, ridicule, nagging, threats, and application of bitter-tasting are not appropriate approaches to manage the patient. The key to success is the nail biter's consent and cooperation. Placebo effect is always better than the punishment in nail biter.

\section{NON-PHARMACOLOGIC TREATMENT}

There are different modalities of non-pharmacologic treatment of NB. Behavioural therapy [23] is the first line gold standard treatment. In the behavioural therapy, various techniques can be used such as nonremovable reminders for NB [24].

- Cognitive behavioural therapy (CBT): It is a common type of psychotherapy, based on cognitive, behavioural, and on learning principles. It is beneficial when simpler measures are not effective. It helps patients to understand that their belief about the behaviour might be incorrect. Later they become aware of negative emotions and related habits so that they can be handle with more effective ways. It is associated with the competing response. Competing response is a method in which a person is provided with an alternative to nail biting options such as chewing gum to satisfy orally-motivated urges.

- Interpersonal psychotherapy (IPT): This psychotherapy based on interpersonal issues so in such type of psychotherapy aim is to improve interpersonal communication and increased social support.

- Dialectic behaviour therapy (DBT): This psychotherapy is conducted with patients with personality disturbances. It is specially made in such a way that to change one's patterns of behaviour that is harmful.

- Habit reversal therapy (HRT): This psychotherapy is based on to chase the habit of nail biting and possibly replaced into more productive habit. It works when the automatic response is weak. It is four-step process teaches a person how to breathe and feel grounded, achieve relaxation, and to complete muscle-response exercises. In this therapy self-control intervention should build selfconfidence and self-esteem [25-26]. This psychotherapy consists of many components such as awareness training; bringing the habit into consciousness, relaxation training, competent response training; engaging in an opposing behaviour that makes it physically impossible to pick or bite until the urge subsides, social support and contingency management.

- Nail cosmetics: Different types of cosmetic used to prevent from nail biting. It helps to enhance nail biting social effects [27].

- Emotional Freedom Technique (EFT): This type of psychotherapy is powerful. Selfhelp technique and an energy therapy are also used to prevent from NB. It has been used and highly effective in addictive habitual behavioural actions and it overcomes unwanted habitual patterns. EFT can help you to stop biting nails by working on the underlying triggers such as stress and others. EFT can be used to install new behaviour as a response to stress, strain or boredom. It works quickly and effectively without any side effects. EFT is commonly used in sports psychology. Sports players are taught how to relieve the stress and boost sports performance. It works as a psychological acupressure. It is based on the same energy meridians used in traditional acupuncture to treat physical and emotional ailments. It is also called tapping method. In this therapy psychotherapist taps different parts of body with finger tips and it is used to input kinetic energy onto specific meridians on the head and chest. Starting tapping from top of head, eyebrow, side of eyes, below nose, chin, collar bone, and under the arm. EFT particularly doesn't work on the nail-biting problem; but it works on the underlying emotional pattern that precedes each bout of nail biting. 
- Aversion stimulus: In this psychotherapy, irritant is used over the finger. This type of psychotherapy is based on reinforcement learning. An aversive stimulus such as a bitter substance on the nail biter's nail so nail biter thinks twice to put nail in mouth [28].

- Stimulus control: The main principle of this psychotherapy is to identify and then eliminate the stimulus; because it always triggers biting urges. This therapy helps to identify, to get rid from environment and situation or emotions that trigger nail biting. The main goal of this therapy is to control triggers through conscious behaviour modification.

Other psychotherapies such as acceptance commitment therapy (ACT) and self- help group are also helpful.

\section{PHARMACOLOGICAL TREATMENT}

If psychotherapy is does not work, particularly in severe cases, selective serotonin reuptake inhibitors like fluoxetine [28] and tricyclic such as clomipramine are the most commonly recommended medications in the management of NB. Lithium is also effective medication resolving nail biting in individuals with a history of bipolar disorder and depressed patient with nail biting [29]. One more drug such as Nacetylcysteine has been used to treat NB but it is with limited efficacy [30].

\section{CONCLUSIONS}

NB is a complex disorder, actually it is not one symptom but it is cluster of symptoms. It is misdiagnosed, ignored condition and poorly understood in clinical practice therefore it may lead to negative effect on quality of life. In such cases we need to educate people, stimulate good habits and develop conscious awareness. It is also very important that patient should be given emotional support and encouragement instead of criticism. Multidisciplinary approach should focus on efforts to build up the child's selfconfidence and self-esteem. Punishment is not effective and its effect is not more than placebo. Behavioural modification techniques and positive reinforcement are important factors during treatment of nail biting.

\section{REFERENCES}

1. Bohne A, Keuthen N, Wilhelm S. Pathologic hairpulling, skin picking, and nail biting. Ann Clin Psychiatry 2005;17(4):227-32.

2. Tanaka OM, Vitral RW, Tanaka GY, Guerrero AP, Camargo ES. Nailbiting, or onychophagia: a special habit. Am J Orthodont Dentofac Orthoped 2008;134(2):305-8.

3. Hatjigiorgis CG, Martin JW. An interim prosthesis to prevent lip and cheek biting. J Prosth Dentistry 1988;59(2):250-2.

4. Lyon LS. A behavioural treatment of compulsive lip-biting. J Behav Ther Exp Psychiatry 1983;14(3):275-6

5. Birch LB. The incidence of nail biting among school children. Br J Educ Psychol 1955;25(2):123-8.

6. Nilner M. Relationships Between Oral Parafunctions and Functional Disturbances and Diseases of the Stomatognathic System Among Children Aged 7-14 Years. Acta Odontol Scand 1983;41(3):167-72.

7. Leung AKC, Robson WLM. Nail Biting. Clin Pediatr 1990;(29)12:690-2.

8. Gregory LH. Stereotypic movement disorder and disorder of infancy, childhood, or adolescence NOS Comprehensive textbook of psychiatry 1995:2360-2.

9. Roberts S, O'Connor K, Aardema F, Bélanger C. The impact of emotions on body-Focused repetitive behaviours: Evidence from a non-treatment-seeking sample. J Behav Ther Exp Psychiatry 2015;46:189-97.

10. Ghanizadeh A. ADHD, bruxism and psychiatric disorders: does bruxism increase the chance of a comorbid psychiatric disorder in children with ADHD and their parents ?. Sleep Breath 2008;12(4):375-80.

11. Halteh P, Scher RK, Lipner SR. Onychophagia: A nail-biting conundrum for physicians. J Dermatol Treat 2017;28(2):166-72.

12. Gilleard E, Eskin M, Savaşir B. Nailbiting and oral aggression in a Turkish student population. Br J Med Psychol 1988;61(2):197-201.

13. Maraz A, Hende B, Urbán R, Demetrovics Z. Pathological grooming: Evidence for a single factor behind trichotillomania, skin picking and nail biting. PLoS One 2017;12(9):e0183806.

14. Foster LG. Nervous habits and stereotyped behaviours in preschool children. J Am Acad Child Adolesc Psychiatry 1998;37(7):711-7.

15. Ghanizadeh A. Association of nail biting and psychiatric disorders in children and their parents in a psychiatrically referred sample of children. Child Adolesc Psychiatr Ment Health 2008;2(1):13-8. 
16. Wells JH, Haines J, Williams CL. Severe morbid onychophagia: the classification as self-mutilation and a proposed model of maintenance. Austr NZ J Psychiatry 1998;32(4):534-45.

17. Lee DY. Chronic nail biting and irreversible shortening of the fingernails. J Eur Acad Dermatol Venereol JEADV 2009;23(2):185-90.

18. Sousa D, Pinto D, Araujo R, Rego RO, Moreira-Neto J. Gingival abscess due to an unusual nail-biting habit: a case report. J Contemp Dent Pract 2010;11(2):85-91.

19. Bello J, Núñez FA, González OM, Fernández R, Almirall P, Escobedo AA. Risk factors for Giardia infection among hospitalized children in Cuba. Ann Trop Med Parasitol 2011;105(1):57-64.

20. Siddiqui JA, Qureshi SF, Marei WM, Mahfouz TA. Onychophagia (Nail biting): A body focused repetitive behaviour due to psychiatric co-morbidity. J Mood Disord 2017;7(1):47.

21. Velazquez L, Ward-Chene L, Loosigian SR. Fluoxetine in the treatment of self-mutilating behaviour. J Am Acad Child Adolesc Psychiatry 2000;39(7):812-4.

22. Leonard HL, Lenane MC, Swedo SE, Rettew DC, Rapoport JL. A double-blind comparison of clomipramine and desipramine treatment of severe onychophagia (nail biting). Arch Gen Psychiatry 1991;48(9):821-7.

23. Ravindran AV, da Silva TL, Ravindran LN, Richter MA, Rector NA. Obsessive-compulsive spectrum disorders: a review of the evidence-based treatments. Can J Psychiatry 2009;54(5):331-43.

24. Koritzky G, Yechiam E. On the value of nonremovable reminders for behaviour modification: an application to nail-biting (onychophagia). Behav Modif 2011;35(6):511-30.

25. Bate KS, Malouff JM, Thorsteinsson ET, Bhullar N. The efficacy of habit reversal therapy for tics, habit disorders, and stuttering: a meta-analytic review. Clin Psychol Rev 2011;31(5):865-71.

26. Ghanizadeh A, Bazrafshan A, Firoozabadi A, Dehbozorgi G. Habit reversal versus object manipulation training for treating nail biting: A randomized controlled clinical trial. Iran J Psychiatry 2013;8(2):61-8.

27. Iorizzo M, Piraccini BM, Tosti A. Nail cosmetics in nail disorders. J Cosmetic Dermatol 2007;6(1):53-8.

28. Silber KP, Haynes CE. Treating nailbiting: a comparative analysis of mild aversion and competing response therapies. Behav Res Ther 1992;30(1):15-22.

29. Sharma V, Sommerdyk C. Lithium treatment of chronic nail biting. Prim Care Comp CNS Disord 2014;16(3)

30. Ghanizadeh A, Derakhshan N, Berk M. N-acetylcysteine versus placebo for treating nail biting, a double blind randomized placebo controlled clinical trial. AntiInflamm AntiAllergy Agents Med Chem 2013;12(3):223-8.

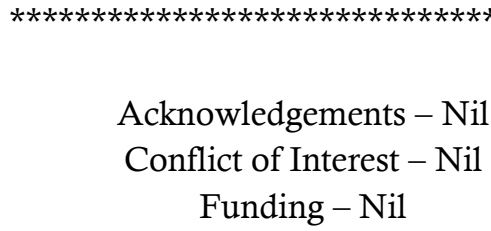

